\title{
Inducing Gaze Gestures by Static Illustrations
}

\author{
Päivi Majaranta \\ paivi.majaranta@tuni.fi \\ Tampere University \\ Tampere, Finland
}

\author{
Jari Laitinen \\ jari.laitinen@sci.fi \\ Tampere University \\ Tampere, Finland
}

\author{
Jari Kangas \\ jari.a.kangas@tuni.fi \\ Tampere University \\ Tampere, Finland
}

\author{
Poika Isokoski \\ poika.isokoski@tuni.fi \\ Tampere University \\ Tampere, Finland
}

\begin{abstract}
In gesture-based user interfaces, the effort needed for learning the gestures is a persistent problem that hinders their adoption in products. However, people's natural gaze paths form shapes during viewing. For example, reading creates a recognizable pattern. These gaze patterns can be utilized in human-technology interaction. We experimented with the idea of inducing specific gaze patterns by static drawings. The drawings included visual hints to guide the gaze. By looking at the parts of the drawing, the user's gaze composed a gaze gesture that activated a command. We organized a proof-of-concept trial to see how intuitive the idea is. Most participants understood the idea without specific instructions already on the first round of trials. We argue that with careful design the form of objects and especially their decorative details can serve as a gaze-based user interface in smart homes and other environments of ubiquitous computing. ${ }^{1}$
\end{abstract}

\section{CCS CONCEPTS}

- Human-centered computing $\rightarrow$ Interaction techniques; Ubiquitous and mobile computing.

\section{KEYWORDS}

Gaze gestures, Gesture-based user interfaces, Gaze-based HCI

\section{INTRODUCTION}

Because we naturally focus our gaze on objects we want to interact with, the gaze is an obvious interaction method in smart environments. However, gaze tracking has some inherent challenges. Pointing with the eyes is fast and easy but accurate gaze pointing requires calibration. That takes time and the calibration may deteriorate over time. Furthermore, using gaze for selection creates a Midas' touch problem where fixated objects are unintentionally selected. Gaze gestures help in overcoming these challenges [Drewes and Schmidt 2007]. They can be recognized by the shape created by the gaze path even from uncalibrated eye tracker data. Learning the mapping between gestures and commands is a problem that reduces the use of gestural user interfaces. More complex gestures are less likely to be confused with natural eye movements [Drewes and Schmidt 2007; Møllenbach et al. 2013] but even more demanding to learn.

We were intrigued by the idea of inducing gaze patterns by illustrations. Instead of asking the user to memorize a specific gaze gesture, we give them something to look at. When looked at in a specific order, the system will interpret that as a command. The idea was inspired by the potential of exploiting people's natural gaze patterns while they observe their environment. Gaze-reactive

\footnotetext{
${ }^{1}$ This is the authors' pre-print. Definitive version available in https://doi.org/10.1145/3317956.3318151
}

objects could be accompanied with visual hints that initiate certain viewing pattern while at the same time making it obvious how to avoid executing the command accidentally. These illustrations could be pieces of art, or stickers on or near the objects that the illustrated gaze gestures control. Having something to look at not only guides the gaze but also avoids the need to perform unnatural, potentially uncomfortable gaze gestures. Avoiding weird looking gaze behavior may be important for the social acceptability of gazebased interaction [Akkil et al. 2016] and affect people's willingness to utilize the technology [Mele and Federici 2012]. Relative gaze gestures can be recognized with electrooculography sensors that are hidden in regular eyeglass frames [Amft et al. 2015] and thus unnoticeable to others.

Below we first briefly review related research. We then introduce the idea and report results from a proof-of-concept study.

\section{RELATED WORK}

Using the sequence of gaze strokes to initiate gaze commands is an old idea. Some of the earliest eye typing systems required the user to glance at a few defined directions in specific order to compose a character [Rosen and Durfee 1978]. Gaze gestures have also been used to control a computer [Porta and Turina 2008], play games [Istance et al. 2010], control mobile phones [Drewes et al. 2007; Kangas et al. 2014] and smart watches [Akkil et al. 2015; Hansen et al. 2016].

Relative gaze gestures are recognized based on the movement pattern and can be done "in the air"; they do not require a target to look at [Bulling et al. 2009; Drewes et al. 2007]. In some implementations, the stroke needs to cross a border or hit a certain area [Huckauf and Urbina 2008; Møllenbach et al. 2013]. These locations serve as hints for the required amplitude and landing position for the stroke. In natural viewing, eye movements are mostly unconscious. Intentionally moving the gaze seems to be easier, if there is a target to look at. For example, in eye drawing task, it is hard to position the eye on a blank canvas but a grid of dots helps to anchor the gaze [Hornof et al. 2004]. The hint for gaze-reactive areas can be given on-screen (see e.g. [Istance et al. 2010]) or off-screen [Isokoski 2000], depending on where the stroke is expected to land.

A visual target is especially useful in smooth pursuit based gaze interactions, where the gaze gesture is performed by following a moving target. Vidal et al. [Vidal et al. 2013] showed that an object can be selected by matching the eye movement path with the object's trajectory. This enables spontaneous interactions in public spaces, without the need for calibration. The idea can be applied in various applications, such as in control of computer widgets [Špakov et al. 2016] or smart watches [Esteves et al. 2015]. In addition to following objects, reading moving text can also be used to initiate commands or calibrate the system [Khamis et al. 2016]. 
It is also possible to guide the gaze using a see-through display to augment the objects with visual hints, as demonstrated by Delamare et al. [Delamare et al. 2017]. Real world objects themselves can be exploited. Jungwirth et al. [Jungwirth et al. 2018] proposed using the object contours to guide gaze gestures. A lamp could be turned on with a gaze gesture composed by tracing the contour of the lampshade. Our proposed method follows the same line of thought, but increases significantly the liberty in choosing gesture shapes by introducing static graphical guides placed on or near the object to be controlled. Furthermore, we only require natural viewing: no prior knowledge of how to perform the gesture is assumed, and there is no need to memorize the gesture.

\section{OUR APPROACH}

We experimented with the idea of inducing specific gaze patterns by static drawings. The drawings include visual hints to guide the gaze. By looking at the parts of the drawing, the user's gaze composes a gaze gesture that activates a command. To separate the gaze gestures from natural viewing, the gesture has to be complex enough. Even single gaze strokes have been used to initiate gaze commands [Møllenbach et al. 2013] but our approach requires several "turning points" (branches) to ensure a distinct pattern.

We ran a few pilot studies to see what kind of illustrations are easy enough for users to work with. These included drawings, text, and symbols. Based on the results from the pilots, we selected a few options for the proof-of-concept test. Simple tree graphs seemed to work best and were easy enough to draw. The nodes in the graph included hints to guide the gaze along the appropriate path of the graph. As hints, we used text reading direction (e.g. turn $\rightarrow$ the $\rightarrow$ light $\rightarrow$ off/on) as well as symbols (e.g. arrows or numbers to indicate the path to be taken, and symbols of lit / unlit lamp). The illustrations are shown in Figure 1.

\section{PROOF-OF-CONCEPT TRIAL}

The main aim of the proof-of-concept trial was to study the intuitiveness of the idea: how easily people understand what to do without instructions, i.e. perform a gaze gesture just by looking at a static drawing. The trial was organized in a laboratory at Tampere University in autumn 2016. The illustrations were shown on a computer screen for convenience.

\subsection{Experimental Software}

For the proof-of-concept trials we implemented a simple gaze gesture recognizer. The gaze tracker was fixed to a regular 19-inch display, i.e. we simplified the setup by making sure that the gaze positions were in the same coordinate system with which the illustrations were drawn. Model gestures were given to the recognizer as a set of sequences of coordinate pairs. The typical transitions between nodes in models (see Figure 1) were 5 to 10 degrees long. The sequence of user's gaze fixations was compared to all models. If the most closely matching model was similar enough to the gaze gesture, the system would trigger a command.

For each gaze sample the system first checked if the current sample was far enough from the previous fixation to potentially create a new fixation. If enough gaze samples formed a new cluster far enough from the previous fixation that cluster location was taken
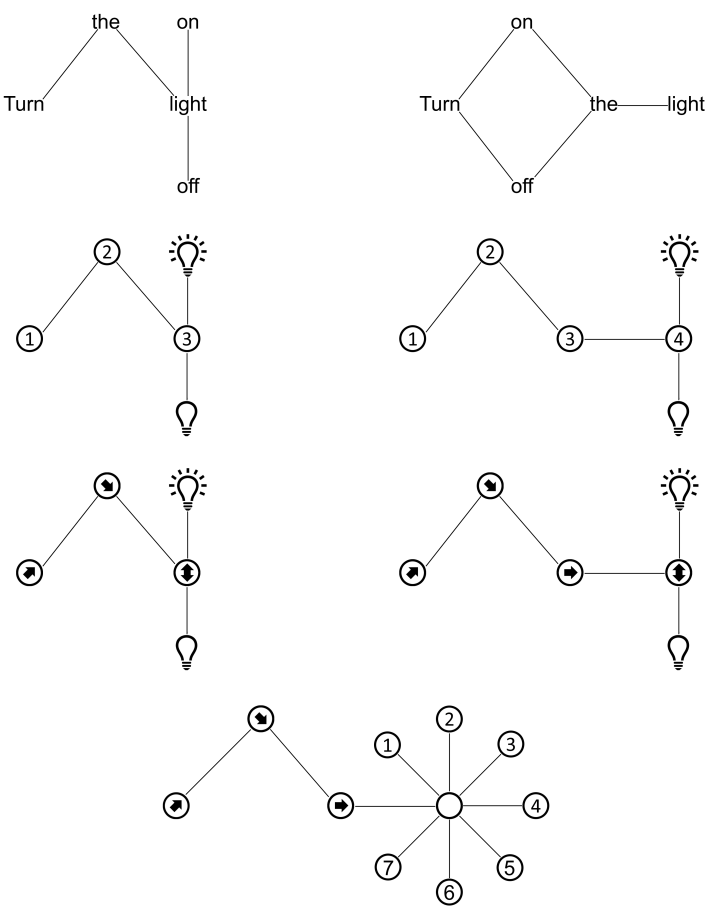

Figure 1: The illustrations used in the experiment.

as the next fixation, and the transition between these two fixations was used in the model comparisons. The model comparison was done always after a new fixation was detected to see if any model was similar enough to the latest fixation sequence.

In our proof-of-concept trials, we used the following parameters: For fixations, we calculated an average of gaze locations over 50 milliseconds (i.e. previous three samples with a $60 \mathrm{~Hz}$ tracker) and then computed whether next gaze sample was closer than 1 degree from that average. If so, we had a potential fixation. There was no dwell-time for fixation detection. For gaze transition calculations, the potential fixation needed to be, at least, 4 degrees away from the previous fixation to be counted as a next fixation. In the gesture model comparison we calculated the average of differences between corresponding transition vectors throughout the length of the gesture as the error term. If the error term was below 3 degrees, we selected the model with smallest error and triggered a command. All these parameters were determined during several pilot user trials. However, as we saw in the proof-of-concept trials, further development and parameter tuning would be beneficial.

\subsection{Setup and Procedure}

The Tobii EyeX tracker was used in the trials. It was pre-calibrated before the experiment by a person who did not participate in the experiment and the same calibration was used throughout the experiment. Thus, the tracker was not calibrated for any of the participants. However, we ensured that tracker saw the participant's eyes by checking the indicator provided by the Tobii EyeX driver. Fourteen participants (10 males, 4 females, aged between 19-34, 
with average age 24 years) volunteered for the experiment. All participants were students of Tampere University. The participants received partial course credit as reward for participation. Two of them had used an eye tracker before. Two wore eye glasses and four wore contact lenses.

Each participant first received a short introduction to the experiment and was then asked to fill in an informed consent form and a background questionnaire. The participant was seated about $70 \mathrm{~cm}$ away from the tracker. She was instructed to read the task (shown on the screen) aloud, indicate she understood it and then act accordingly. Tasks were done by looking at the screen; no mouse or keyboard was used. The participant could skip a task or ask questions but the researcher did not provide help during the tasks.

There were two types of tasks: (1) turning the light on or off, and (2) ordering an elevator to a specified floor (floors 1-7). For turning the light on or off, there were six different illustrations used as hints for the gaze gesture (see Figure 1). To order the elevator, we used the illustration also shown in Figure 1. If the task was successful, i.e. the participant was able to do a gaze gesture, the participant received a written note on the screen (e.g. "you turned on the light", "you ordered the elevator to floor 3"). In the case of failure, nothing happened. Participants were given at least two minutes to try completing the task before asking if they wanted to skip to the next one.

The experiment had two phases. The purpose of phase 1 was to see how well the participants can use the system without instruction. Phase 1 included 13 trials. 12 of the trials concerned turning the light on (6 trials) and then off ( 6 trials). Order was balanced by Latin square. In addition, there was one trial to order the lift to the given floor. With 14 participants and 7 possible floors, each floor number was ordered twice during the first phase in random order

The purpose of phase 2 was to collect some initial performance data on the use of the system. Before the phase 2 started, the partic ipant was asked whether she feels like she knew how the system worked. Regardless of the answer, short instructions were given Phase 2 included 50 trials. The order of the tasks was same as in phase 1 , but each of the six illustrations for lights was repeated three times so that the participant first turned the light on and then off ( 6 trials). The lift was ordered to each floor twice. The order was balanced by Latin square. In total, each participant performed 63 trials.

In the end of both phases, the participant was asked to fill in a questionnaire. In the end of the session, the participant was interviewed. In total, the experiment took one hour or less.

\subsection{Results}

In phase 1 the participants were not given any instructions on how to do the given tasks and we were mostly interested to see if the participants were able to realize how the system was to be used. If the participant didn't figure it out s/he was always allowed to skip a task and proceed to the next one.

All but one (13/14) of the participants figured out how to do the gestures during phase 1 and were confidently completing the tasks. Nine participants (9/14) didn't skip any of the tasks. Four participants (4/14) skipped between 1 and 4 tasks, while one participant skipped 11 out of 13 tasks.
In phase 2, after the gesture system was explained to all participants, no tasks were skipped, which shows the power of training. We intentionally didn't train the participants for the first session to see whether the illustrations contain enough hints without explicit instructions.

We measured the completion time for all participants and all tasks. In the order of presentation during the phase 1 there was a clear learning effect, see Figure 2 . The mean value for the first task was 25 seconds but the mean completion time approached 5 seconds already around task number 5 . Throughout phase 2 the mean completion time was around 4-5 seconds.

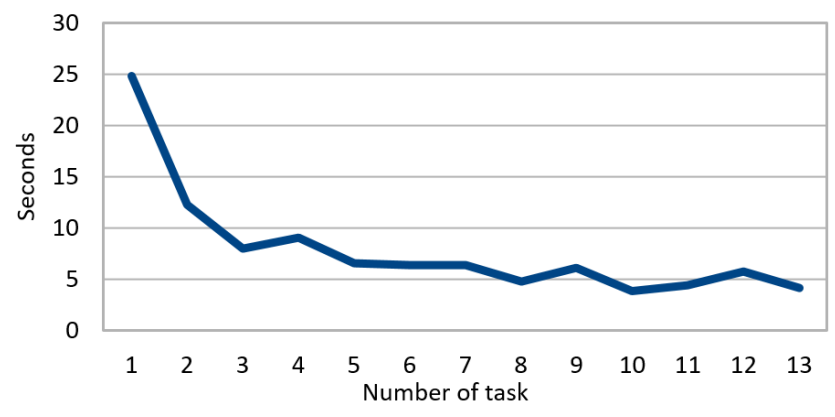

Figure 2: The mean task completion times in the order of task presentation for phase 1 .

Overall, around $1 / 20$ of the gesture commands were erroneous (i.e. wrong gesture was recognized). In phase 1 some errors probably happened because the participants didn't fully understand the idea. In phase 2 most of the errors happened in the elevator task, which required more accurate separation between different gestures. There were errors also in the lamp tasks, but in most cases the participants indicated that they had forgotten the task, or made other mistakes. Many (9/14) participants said that there were instances where they thought they had made the correct gesture, but that the recognizer did not seem to recognize it. The number of recognizer mistakes could be decreased by more careful algorithm development and parameter tuning as well as utilizing different gestures. E.g. in the elevator case the diagonal numbers could be further from the hub to increase separation.

After each phase, the participant filled in a semantic differential rating scale questionnaire about the understandability and pleasantness of each illustration, and the participant's perceived success in the task. Overall, the ratings were rather positive. In the 7-step scale (from 1 positive to 7 negative), the average ratings for all illustrations were below 3 in both phases: 1.9 and 1.7 (understandability); 2.2 and 2.0 (pleasantness); 1.9 and 2.1 (perceived success), in phase 1 and 2, respectively. The elevator got the worst average ratings in all categories in both phases. However, the differences were quite small. For example, in phrase 1, the average ratings for the understandability of the illustrations varied between 1.4 (arrow icons for lamp on/off) and 2.6 (elevator).

In the post-test semi-structured interview, we asked about participants' overall impressions, and if they had understood the idea and what they were supposed to do. Participants commented that the 
system was interesting and worked surprisingly well. They understood the idea "immediately", "soon" or "easily". Some participants had tried "sliding" the gaze along the lines, or were unsure how long they need to look at each step. Seven participants commented that they liked the icon-based illustrations more than text based which were preferred by five participants. Some participants felt that reading text was slower than glancing an icon. However, not all participants explained why they liked a certain illustration type more than the other. Related to the problems, participants commented on the need to check the illustration before action to avoid wrong turn in the end, and the need to carefully look at the intended floor number to avoid false selections.

\section{DISCUSSION}

We experimented with the idea of inducing specific gaze patterns by drawings. The proof-of-concept trials show that all but one participant got the idea very early in the experiment and all were able to use the system after a brief explanation. After learning, performing the gaze gestures was quite consistent and robust without the need to calibrate the eye tracker.

Completing a gaze gesture took about $4-5$ seconds. The fastest task completion times were between 1 and 2 seconds. The difference is because participants took some time to orient to the task and in some cases one or more than one retry was needed. Also, we didn't instruct to aim for maximum speed. Even if a command by gaze gesture would be slower than pushing a button, there is use for gaze. Gaze-based environmental control is useful for people with disabilities [Bates et al. 2012]. There are also environments where avoiding physical contact is beneficial, for example, to avoid infections. In our design we are mainly thinking of future living and working environments where many things are connected to the internet. Then building physical user interfaces is an unnecessary expense. On the other hand, being within hand's reach is not needed for operating devices because they can be commanded through the internet.

The performance of the system can be improved by fine-tuning the illustrations. Some types of gestures may be easier to perform than others, and/or be easier to recognize. For example, diagonal gaze strokes may induce more errors than horizontal strokes [Istance and Hyrskykari 2017]. In situations, which require many choices to be made, we could vary the length of the branches or continue the gestures hierarchically when the space on the surface allows this.

We did not give any feedback for unsuccessful gesture recognition. This is because the system does not know when the user is attempting to give a command. It is receiving gaze data all the time and only reacts when it recognizes a pattern that is very close to one of the predefined gesture patterns. A different design could make the user signal that an attempt for a gesture begins, e.g., with speech. The system could also detect that the user is viewing a gesture illustration, for example, from a visual tag that the system recognizes with a forward-looking camera. If the eye tracker is embedded in the environment or the controlled object [Shell et al. 2003; Vidal et al. 2013], it could detect the eyes of the user facing it. It would be useful to give some indication of typical failures, starting from the indication if the tracker sees the eye and if it is in recognition mode. However, we believe that by improving the gesture shapes and recognition technology, we can get to a point where recognition failures that seem unexplainable to the user are rare enough not to be a problem.

The size of the illustration and its distance from the eyes affects its visibility to the user and the magnitude of the eye movements. In our experiment, with the user being about $70 \mathrm{~cm}$ from the display, we required the distance between fixation points to be at least 4 degrees apart from each other. A closer look at the data shows that there were a number of unrecognized gestures that were near the threshold and would have been accepted with a different threshold. Further research is required to find appropriate limits in real life scenarios for robust gaze gesture recognition (depending on the tracker), and, what size of illustration is comfortable for the user to view (depending on the user).

The aesthetics of the illustration should also be considered. We used simple tree graphs for convenience. At home or office, people may prefer more artistic pictures that are in harmony with the environment. Creating such art requires skills and resources. Nevertheless, apart from the design, adding the illustrations is easy and cheap (no electronics required). The illustrations are robust and durable. Creating new, custom gestures is also easy: draw the pictorial hint, gaze it, and record the path to teach a new gesture.

\section{REFERENCES}

Deepak Akkil, Jari Kangas, Jussi Rantala, Poika Isokoski, Oleg Spakov, and Roope Raisamo. 2015. Glance Awareness and Gaze Interaction in Smartwatches. In Proceedings of the 33rd Annual ACM Conference Extended Abstracts on Human Factors in Computing Systems (CHI EA '15). ACM, New York, NY, USA, 1271-1276. https://doi.org/10.1145/2702613.2732816

Deepak Akkil, Andrés Lucero, Jari Kangas, Tero Jokela, Marja Salmimaa, and Roope Raisamo. 2016. User Expectations of Everyday Gaze Interaction on Smartglasses. In Proceedings of the 9th Nordic Conference on Human-Computer Interaction (NordiCHI '16). ACM, New York, NY, USA, Article 24, 10 pages. https://doi.org/10.1145/ 2971485.2971496

Oliver Amft, Florian Wahl, Shoya Ishimaru, and Kai Kunze. 2015. Making regular eyeglasses smart. IEEE Pervasive Computing 14, 3 (2015), 32-43. https://doi.org/10. 1109/MPRV.2015.60

Richard Bates, Emiliano Castellina, Fulvio Corno, Petr Novák, and Olga Štepánková. 2012. Beyond Communication and Control: Environmental Control and Mobility by Gaze. In In Gaze Interaction and Applications of Eye Tracking: Advances in Assistive Technologies, Päivi Majaranta Majaranta, Hirotaka Aoki, Mick Donegan, Dan Witzner Hansen, John Paulin Hansen, Aulikki Hyrskykari, and Kari-Jouko Räihä (Eds.). 103-127.

Andreas Bulling, Daniel Roggen, and Gerhard Tröster. 2009. Wearable EOG Goggles: Eye-based Interaction in Everyday Environments. In CHI '09 Extended Abstracts on Human Factors in Computing Systems (CHI EA '09). ACM, New York, NY, USA, 3259-3264. https://doi.org/10.1145/1520340.1520468

William Delamare, Teng Han, and Pourang Irani. 2017. Designing a Gaze Gesture Guiding System. In Proceedings of the 19th International Conference on HumanComputer Interaction with Mobile Devices and Services (MobileHCI '17). ACM, New York, NY, USA, Article 26, 13 pages. https://doi.org/10.1145/3098279.3098561

Heiko Drewes, Alexander De Luca, and Albrecht Schmidt. 2007. Eye-gaze interaction for mobile phones. In Proceedings of the 4th international conference on mobile technology, applications, and systems and the 1st international symposium on Computer human interaction in mobile technology. ACM, 364-371.

Heiko Drewes and Albrecht Schmidt. 2007. Interacting with the computer using gaze gestures. In IFIP Conference on Human-Computer Interaction. Springer, 475-488.

Augusto Esteves, Eduardo Velloso, Andreas Bulling, and Hans Gellersen. 2015. Orbits: Gaze Interaction for Smart Watches Using Smooth Pursuit Eye Movements. In Proceedings of the 28th Annual ACM Symposium on User Interface Software \& Technology (UIST '15). ACM, New York, NY, USA, 457-466. https://doi.org/10.1145/ 2807442.2807499

John Paulin Hansen, Haakon Lund, Florian Biermann, Emillie Møllenbach, Sebastian Sztuk, and Javier San Agustin. 2016. Wrist-worn pervasive gaze interaction. In Proceedings of the ninth biennial ACM symposium on eye tracking research \& applications. ACM, 57-64. 
Anthony Hornof, Anna Cavender, and Rob Hoselton. 2004. Eyedraw: A System for Drawing Pictures with Eye Movements. In Proceedings of the 6th International ACM SIGACCESS Conference on Computers and Accessibility (Assets '04). ACM, New York, NY, USA, 86-93. https://doi.org/10.1145/1028630.1028647

Anke Huckauf and Mario H. Urbina. 2008. On object selection in gaze controlled environments. Fournal of Eye Movement Research 2, 4 (Nov. 2008). https://doi.org/ 10.16910/jemr.2.4.4

Poika Isokoski. 2000. Text Input Methods for Eye Trackers Using Off-screen Targets. In Proceedings of the 2000 Symposium on Eye Tracking Research \& Applications (ETRA '00). ACM, New York, NY, USA, 15-21. https://doi.org/10.1145/355017.355020

Howell Istance, Aulikki Hyrskykari, Lauri Immonen, Santtu Mansikkamaa, and Stephen Vickers. 2010. Designing Gaze Gestures for Gaming: An Investigation of Performance. In Proceedings of the 2010 Symposium on Eye-Tracking Research \&\#38; Applications (ETRA '10). ACM, New York, NY, USA, 323-330. https: //doi.org/10.1145/1743666.1743740

Howell Istance and Aulikki I. Hyrskykari. 2017. Supporting Making Fixations and the Effect on Gaze Gesture Performance. In Proceedings of the 2017 CHI Conference on Human Factors in Computing Systems (CHI '17). ACM, New York, NY, USA, 3022-3033. https://doi.org/10.1145/3025453.3025920

Florian Jungwirth, Michael Haslgrübler, and Alois Ferscha. 2018. Contour-guided Gaze Gestures: Using Object Contours As Visual Guidance for Triggering Interactions. In Proceedings of the 2018 ACM Symposium on Eye Tracking Research \& Application (ETRA '18). ACM, New York, NY, USA, Article 28, 10 pages. https://doi.org/10. $1145 / 3204493.3204530$

Jari Kangas, Deepak Akkil, Jussi Rantala, Poika Isokoski, Päivi Majaranta, and Roope Raisamo. 2014. Gaze Gestures and Haptic Feedback in Mobile Devices. In Proceedings of the SIGCHI Conference on Human Factors in Computing Systems (CHI '14). ACM, New York, NY, USA, 435-438. https://doi.org/10.1145/2556288.2557040
Mohamed Khamis, Ozan Saltuk, Alina Hang, Katharina Stolz, Andreas Bulling, and Florian Alt. 2016. TextPursuits: Using Text for Pursuits-based Interaction and Calibration on Public Displays. In Proceedings of the 2016 ACM International foint Conference on Pervasive and Ubiquitous Computing (UbiComp '16). ACM, New York, NY, USA, 274-285. https://doi.org/10.1145/2971648.2971679

Maria Laura Mele and Stefano Federici. 2012. A psychotechnological review on eyetracking systems: towards user experience. Disability and Rehabilitation: Assistive Technology 7, 4 (2012), 261-281.

Emilie Møllenbach, John Paulin Hansen, and Martin Lillholm. 2013. Eye movements in gaze interaction. Journal of Eye Movement Research 6, 2 (2013), 1-15.

Marco Porta and Matteo Turina. 2008. Eye-S: a full-screen input modality for pure eye-based communication. In Proceedings of the 2008 symposium on Eye tracking research \& applications. ACM, 27-34.

Michael J. Rosen and William K. Durfee. 1978. Preliminary report on eyecom. In Proceedings 5th Annual Conference on Systems and Devices for the Disabled. 167.

Jeffrey S. Shell, Roel Vertegaal, and Alexander W. Skaburskis. 2003. EyePliances: Attention-seeking Devices That Respond to Visual Attention. In CHI '03 Extended Abstracts on Human Factors in Computing Systems (CHI EA '03). ACM, New York, NY, USA, 770-771. https://doi.org/10.1145/765891.765981

Oleg Špakov, Poika Isokoski, Jari Kangas, Deepak Akkil, and Päivi Majaranta. 2016. PursuitAdjuster: An Exploration into the Design Space of Smooth Pursuit -based Widgets. In Proceedings of the Ninth Biennial ACM Symposium on Eye Tracking Research \& Applications (ETRA '16). ACM, New York, NY, USA, 287-290. https: //doi.org/10.1145/2857491.2857526

Mélodie Vidal, Andreas Bulling, and Hans Gellersen. 2013. Pursuits: Spontaneous Interaction with Displays Based on Smooth Pursuit Eye Movement and Moving Targets. In Proceedings of the 2013 ACM International foint Conference on Pervasive and Ubiquitous Computing (UbiComp '13). ACM, New York, NY, USA, 439-448. https://doi.org/10.1145/2493432.2493477 